\title{
Frequency formula for a class of fractal vibration system
}

\author{
Yi Tian ${ }^{1,2}$ \\ ${ }^{1}$ College of Data Science and Application, Inner Mongolia University of Technology, Hohhot, China, e-mail: \\ ttxsun@163.com; \\ ${ }^{2}$ Inner Mongolia Autonomous Region Engineering and Technology Research Center of Big Data Based \\ Software Service, Hohhot, China
}

\begin{tabular}{|c|c|}
\hline Article Info & ABSTRACT \\
\hline Article history: & \multirow{5}{*}{$\begin{array}{l}\text { Four fractal nonlinear oscillators (The fractal Duffing oscillator, fractal } \\
\text { attachment oscillator, fractal Toda oscillator, and a fractal nonlinear oscillator) } \\
\text { are successfully established by He's fractal derivative in a fractal space, and } \\
\text { their variational principles are obtained by semi-inverse transform method. } \\
\text { The approximate frequency of the four fractal oscillators are found by a simple } \\
\text { frequency formula. The results show the frequency formula is a powerful and } \\
\text { simple tool to a class of fractal oscillators. }\end{array}$} \\
\hline Received October 19, 2021 & \\
\hline Revised December 27, 2021 & \\
\hline Accepted January 1, 2022 & \\
\hline & \\
\hline
\end{tabular}

Fractal oscillator,

He's fractal derivative,

Variational principle,

Semi-inverse transform method.

Copyright (C) 2022 Regional Association for Security and crisis management and European centre for operational research. All rights reserved.

\section{Corresponding Author:}

Yi Tian,

College of Data Science and Application, Inner Mongolia University of Technology, Hohhot, China.

Email: ttxsun@163.com

\section{Introduction}

The partial differential equations (PDEs) arise in many fields like the condense matter physics, fluid mechanics, economics and management, etc. There are many methods for solving nonlinear PDEs, for example, the homotopy perturbation method (Anjum \& He, 2020a, 2020b; He, 2003; He \& El-Dib, 2020; Yu, et al., 2019), variational iteration method (Anjum \& He, 2019; He, 1999), Taylor series method (He, 2019,2020a; He \& Ji, 2019a; He, et al., 2020), Exp-function method (He, 2013; He \& Wu, 2006), and variational-based methods (He, 2020b,2021; He \& Ai, 2020). In this paper, we maily study on a class of vibration equation (He, et al., 2021a)

$m w^{\prime \prime}+h(w)=0$

with the initial condition

$w(0)=a, w^{\prime}(0)=b$

where $m$ is the mass, $h$ is the nonlinear restoring force, and $a$ and $b$ are constants.

Vibration is the intrinsic property of a packing system, and so far there is no way to stop the vibration, the frequency-amplitude is the main factor for designing a packing system (Song, 2020). The frequency formulation for oscillator (1) was proposed as (He, et al., 2021a) 
$\omega=\left.\sqrt{\frac{h(w)}{w}}\right|_{w=N A}$

where $A$ is the amplitude, it can be approximated calculated as

$A=\sqrt{a^{2}+\frac{b^{2}}{\omega^{2}}}$

and $N$ is $\sqrt{3} / 2$ for non-singular oscillators and 0.8 for singular oscillators.

\section{Fractal Duffing oscillator}

Consider the following well-known Duffing oscillator (He, et al., 2021b)

$\frac{d^{2} w}{d t^{2}}+w+\varepsilon w^{3}=0$

with the initial condition

$w(0)=A, w^{\prime}(0)=0$

\section{Variational principle of fractal Duffing oscillator}

In a fractal space, Eq. (5) can be described by He's fractal derivative as follows

$\frac{{ }^{H} D}{D t^{\alpha}}\left(\frac{{ }^{H} D w}{D t^{\alpha}}\right)+w+\varepsilon w^{3}=0$

with the initial condition

$w(0)=A, \frac{{ }^{H} D w(0)}{D t^{\alpha}}=0$

where ${ }^{H} D w / D t^{\alpha}$ is He's fractal derivative and is defined as follows (He,2018)

$\frac{{ }^{H} D w}{D t^{\alpha}}\left(t_{0}\right)=\Gamma(1+\alpha) \lim _{\substack{t-t_{\rightarrow} \rightarrow \Delta t \\ \Delta t \neq 0}} \frac{w(t)-w\left(t_{0}\right)}{\left(t-t_{0}\right)^{\alpha}}$

The variational principle (He, 2020c) of Eq. (7) can be given by semi-inverse transform method as follows

$J(w)=\int\left\{\frac{1}{2}\left(\frac{{ }^{H} D w}{D t^{\alpha}}\right)^{2}-\frac{1}{2} w^{2}-\frac{1}{4} \varepsilon w^{4}\right\}{ }^{H} D t^{\alpha}$

\section{Fractal frequency formula}

Using the two-scale transform method (He \& Ji, 2019b) to Eq. (7) and assume

$T=t^{\alpha}$

therefore, Eq. (7) can be written as follows

$\frac{{ }^{H} D}{D T}\left(\frac{{ }^{H} D w}{D T}\right)+w+\varepsilon w^{3}=0$

with the initial condition 
$w(0)=A, \frac{{ }^{H} D w(0)}{D T}=0$

Adopt the frequency formula (3), and the approximate frequency can be easily obtained as follows

$\omega=\left.\sqrt{\frac{w+\varepsilon w^{3}}{w}}\right|_{w=\frac{\sqrt{3}}{2} A}=\sqrt{1+\frac{3}{4} \varepsilon A^{2}}$

Table 1. Comparison of Eq. (14) with Eq. (24) in (He, et al., 2021b)

\begin{tabular}{cccccccc}
\hline$\varepsilon A^{2}$ & 0 & 0.001 & 0.0025 & 0.003 & 0.005 & 0.007 & 0.009 \\
\hline $\begin{array}{c}\text { Eq.(14) } \\
\text { Eq.(24) in (He,et } \\
\text { al. 2021b) }\end{array}$ & 1 & 1.000375 & 1.0009371 & 1.001124 & 1.0018724 & 1.002622 & 1.003369 \\
Exact frequency & 1 & 1.000380 & 1.0009442 & 1.00113 & 1.0018726 & 1.002613 & 1.003369 \\
\hline
\end{tabular}

Remark 1.

The Eq. (14) gives more accurate results than Eq.(24) in (He, et al., 2021b) for the fractal Duffing oscillator.

\section{Fractal attachment oscillator}

Consider the following attachment oscillator (Ren, et al., 2019)

$\frac{d^{2} u}{d t^{2}}+\frac{\varepsilon}{u^{3}}=0$

with the initial condition

$u(0)=A, u^{\prime}(0)=0$

\section{Variational principle of fractal attachment oscillator}

In a fractal space, Eq. (15) can be described by He's fractal derivative as follows

$\frac{{ }^{H} D}{D t^{\alpha}}\left(\frac{{ }^{H} D u}{D t^{\alpha}}\right)+\frac{\varepsilon}{u^{3}}=0$

with the initial condition

$u(0)=A, \frac{{ }^{H} D u(0)}{D t^{\alpha}}=0$

where ${ }^{H} \mathrm{Du} / \mathrm{Dt}{ }^{\alpha}$ is He's fractal derivative and is defined as follows

$\frac{{ }^{H} D u}{D t^{\alpha}}\left(t_{0}\right)=\Gamma(1+\alpha) \lim _{\substack{t-t_{0} \rightarrow \Delta t \\ \Delta \neq 0}} \frac{u(t)-u\left(t_{0}\right)}{\left(t-t_{0}\right)^{\alpha}}$

The variational principle of Eq. (17) can be given by semi-inverse transform method as follows

$J(u)=\int\left\{\frac{1}{2}\left(\frac{{ }^{H} D u}{D t^{\alpha}}\right)^{2}+\frac{\varepsilon}{2 u^{2}}\right\}{ }^{H} D t^{\alpha}$ 


\section{Fractal frequency formula}

Using the two-scale transform method to Eq. (17) and assume

$T=t^{\alpha}$

therefore, Eq. (17) can be written as follows

$\frac{{ }^{H} D}{D T}\left(\frac{{ }^{H} D u}{D T}\right)+\frac{\varepsilon}{u^{3}}=0$

with the initial condition

$u(0)=A, \frac{{ }^{H} D u(0)}{D T}=0$

Adopt the fractal frequency formula (3), and the approximate frequency can be easily obtained as follows

$\omega=\left.\sqrt{\frac{\varepsilon}{u^{4}}}\right|_{u=0.8 \mathrm{~A}}=1.5625 \sqrt{\frac{\varepsilon}{A^{4}}}$

\section{Remark 2.}

In (Ren,et al., 2019), the explicit form of the frequency formula of attachment oscillator is not given

\section{Fractal Toda oscillator}

Consider the following Toda oscillator (He, et al., 2021c)

$\frac{d^{2} u}{d t^{2}}+e^{u}-1=0$

with the initial condition

$u(0)=A, u^{\prime}(0)=0$

\section{Variational principle of fractal Toda oscillator}

In a fractal space, Eq. (25) can be described by He's fractal derivative as follows

$\frac{{ }^{H} D}{D t^{\alpha}}\left(\frac{{ }^{H} D u}{D t^{\alpha}}\right)+e^{u}-1=0$

with the initial condition

$u(0)=A, \frac{{ }^{H} D u(0)}{D t^{\alpha}}=0$

where ${ }^{H} D u / D t^{\alpha}$ He's fractal derivative and is defined as follows

$\frac{{ }^{H} D u}{D t^{\alpha}}\left(t_{0}\right)=\Gamma(1+\alpha) \lim _{\substack{t-t_{0} \rightarrow \Delta t \\ \Delta t \neq 0}} \frac{u(t)-u\left(t_{0}\right)}{\left(t-t_{0}\right)^{\alpha}}$

The variational principle of Eq. (27) can be given by semi-inverse transform method as follows

$J(u)=\int\left\{\frac{1}{2}\left(\frac{{ }^{H} D u}{D t^{\alpha}}\right)^{2}+e^{u}-u\right\}{ }^{H} D t^{\alpha}$ 


\section{Fractal frequency formula}

Using the two-scale transform method to Eq. (27) and assume

$T=t^{\alpha}$

therefore, Eq. (27) can be written as follows

$$
\frac{{ }^{H} D}{D T}\left(\frac{{ }^{H} D u}{D T}\right)+e^{u}-1=0
$$

with the initial condition

$$
u(0)=A, \frac{{ }^{H} D u(0)}{D T}=0
$$

Adopt the frequency formula (3), and the approximate frequency can be easily obtained as follows

$\omega=\left.\sqrt{\frac{e^{u}-1}{u}}\right|_{u=\frac{\sqrt{3}}{2} A}=\frac{2\left(-1+e^{\frac{\sqrt{3} A}{2}}\right)}{\sqrt{3} A}$

\section{Remark 3.}

In (He, et al., 2021c), the frequency formula (34) is not given for Toda oscillator.

\section{A fractal nonlinear oscillator}

Consider the following nonlinear oscillator (He, 2014)

$$
\frac{d^{2} u}{d t^{2}}+u+\varepsilon_{1} u^{2}+\varepsilon_{2} u^{3}=0
$$

with the initial condition

$u(0)=A, u^{\prime}(0)=0$

\section{Variational principle of a fractal nonlinear oscillator}

In a fractal space, Eq. (35) can be described by He's fractal derivative as follows

$$
\frac{{ }^{H} D}{D t^{\alpha}}\left(\frac{{ }^{H} D u}{D t^{\alpha}}\right)+u+\varepsilon_{1} u^{2}+\varepsilon_{2} u^{3}=0
$$

with the initial condition

$$
u(0)=A, \frac{{ }^{H} D u(0)}{D t^{\alpha}}=0
$$

where ${ }^{H} \mathrm{Du} / \mathrm{D} t^{\alpha} \mathrm{He}$ 's fractal derivative and is defined as follows

$$
\frac{{ }^{H} D u}{D t^{\alpha}}\left(t_{0}\right)=\Gamma(1+\alpha) \lim _{\substack{t-t_{0} \rightarrow \Delta t \\ \Delta t \neq 0}} \frac{u(t)-u\left(t_{0}\right)}{\left(t-t_{0}\right)^{\alpha}}
$$

The variational principle of Eq. (37) can be given by semi-inverse transform method as follows

$$
J(u)=\int\left\{\frac{1}{2}\left(\frac{{ }^{H} D u}{D t^{\alpha}}\right)^{2}-\frac{1}{2} u^{2}+\frac{1}{3} \varepsilon_{1} u^{3}-\frac{1}{4} \varepsilon_{2} u^{4}\right\}{ }^{H} D t^{\alpha}
$$




\section{Fractal frequency formula}

Using the two-scale transform method to Eq. (37) and assume

$T=t^{\alpha}$

therefore, Eq. (37) can be written as follows

$$
\frac{{ }^{H} D}{D T}\left(\frac{{ }^{H} D u}{D T}\right)+u+\varepsilon_{1} u^{2}+\varepsilon_{2} u^{3}=0
$$

with the initial condition

$u(0)=A, \frac{{ }^{H} D u(0)}{D T}=0$

Adopt the frequency formula (3), and the approximate frequency can be easily obtained as follows

$\omega=\left.\sqrt{1+\varepsilon_{1} u+\varepsilon_{2} u^{2}}\right|_{u=\frac{\sqrt{3}}{2} A}=\sqrt{1+\frac{\sqrt{3}}{2} A \varepsilon_{1}+\frac{3}{4} A^{2} \varepsilon_{2}}$

We write down Nayfeh's result (He, 2014) for comparison

$\omega=1+\left(\frac{3}{8} \varepsilon_{2}-\frac{5}{12} \varepsilon_{1}^{2}\right) A^{2}$

Table 2. Comparison of Eq. (44) with Eq. (45)

\begin{tabular}{cccccc}
\hline$A$ & $\varepsilon_{1}$ & $\varepsilon_{2}$ & Eq.(44) & Eq.(45) & Relative error \\
\hline 1 & 0.001 & 0.001 & 1.00081 & 1.00037 & $0.044 \%$ \\
1 & 0.01 & 0.05 & 1.02282 & 1.01871 & $0.4 \%$ \\
10 & 0.0001 & 0.0005 & 1.019 & 1.01875 & $0.025 \%$ \\
10 & 0.001 & 0.0025 & 1.09369 & 1.09371 & $0.0018 \%$ \\
100 & 0.01 & 0.0005 & 2.36982 & 2.45833 & $3.7 \%$ \\
100 & 0.005 & 0.00025 & 1.81879 & 1.83333 & $0.8 \%$ \\
\hline
\end{tabular}

\section{Remark 4}

Table 2 shows the good agreement between Eq. (44) and Eq. (45).

\section{Conclusions}

In this paper, four nonlinear oscillators are described by He's fractal derivative in a fractal space, and their variational principle are successfully established via semi-inverse transform method. The two-scale transform method and fractal frequency formulas are adopted to find the approximate frequency of fractal oscillator equation. The examples show the frequency formula is simple and effective.

\section{References}

Anjum, N., \& He, J.H. (2019). Laplace transform: Making the variational iteration method easier. Applied Mathematics Letters, 92, 134-138.

Anjum, N., \& He, J.H. (2020a). Higher-order homotopy perturbation method for conservative nonlinear oscillators generally and microelectromechanical systems' oscillators particularly. International Journal of Modern Physics B, 34(32), 2050313.

Anjum, N., \&He, J.H. (2020b) Homotopy perturbation method for N/MEMS oscillators. Mathematical Methods in the Applied Sciences, DOI: 10.1002/mma.6583. 
He, C.H., Shen, Y., Ji, F.Y., \& He, J.H. (2020). Taylor series solution for fractal Bratu-type equation arising in electrospinning process. Fractals, 28(1), 2050011.

He, J.H. (1999). Variational iteration method-a kind of non-linear analytical technique: Some examples. International Journal of Non-Linear Mechanics, 34(4), 699-708.

He, J.H. (2003). Homotopy perturbation method: a new nonlinear analytical technique. Applied mathematics and computation, 135(1), 73-79.

He, J.H. (2013). Exp-function method for fractional differential equations. International Journal of Nonlinear Sciences and Numerical Simulation, 14(6), 363-366.

He, J.H. (2014). Homotopy perturbation method with two expanding parameters. Indian Journal of Physics, 88(2), 193-196.

He, J.H. (2018). Fractal calculus and its geometrical explanation. Results in Physics, 10, 272-276.

He, J.H. (2019). A simple approach to one-dimensional convection-diffusion equation and its fractional modification for E reaction arising in rotating disk electrodes. Journal of Electroanalytical Chemistry, 854, 113565.

He, J.H. (2020a). Taylor series solution for a third order boundary value problem arising in architectural engineering. Ain Shams Engineering Journal, 11(4), 1411-1414.

He, J.H. (2020b).Variational principle and periodic solution of the Kundu-Mukherjee-Naskar equation. Results in Physics, 17, 103031.

He, J.H. (2020c).Generalized variational principles for buckling analysis of circular cylinders. Acta Mechanica, 231(3), 899-906.

He, J.H. (2021). On the fractal variational principle for the telegraph equation. Fractals, 29(1), 2150022.

He, J.H., \& Ain, Q.T. (2020). New promises and future challenges of fractal calculus: From two-scale Thermodynamics to fractal variational principle. Thermal Science, 24(2A), 659-681.

He, J.H., \& El-Dib, Y. O. (2020). Homotopy perturbation method for Fangzhu oscillator. Journal of Mathematical Chemistry, 58(10), 2245-2253.

He, J.H., Yang, Q., He, C.H., \& Khan, Y. (2021a). A simple frequency formulation for the tangent oscillator. Axioms, 10,320.

He, J.H., Hou, W.F.,Qie, N.,Gepreel, K. A., Shirazi, A.H., \& Mohammad-Sedighi,H. (2021b). Hamiltonianbased frequency-amplitude formulation for nonlinear oscillators. Facta Universitatis, Series: Mechanical Engineering, 19(2), 199-208.

He, J.H., El-Dib, Y. O., \& Mady, A.A. (2021c). Homotopy perturbation method for the fractal Toda oscillator. Fractal and Fractional, 5, 93.

He, J.H., \& Ji, F.Y. (2019a). Taylor series solution for Lane-Emden equation. Journal of Mathematical Chemistry, 57(8), 1932-1934.

He, J.H., \& Ji, F.Y. (2019b).Two-scale mathematics and fractional calculus for thermodynamics. Thermal Science, 23(4), 2131-2133.

He, J.H., \& Wu, X.H. (2006). Exp-function method for nonlinear wave equations. Chaos Solitons \& Fractals, 30(3), 700-708.

Ren, Z.F., Yao, S.W., \& He, J.H. (2019). He's multiple scales method for nonlinear vibrations. Journal of Low Frequency Noise Vibration and Active Control, 38(3-4), 1708-1712.

Song, H.Y. (2020). A thermodynamic model for a packing dynamical system. Thermal Science, 24(4), 23312335 .

Yu, D.N., He, J.H., \& Garcia, A. G. (2019). Homotopy perturbation method with an auxiliary parameter for nonlinear oscillators. Journal of Low Frequency Noise Vibration and Active Control, 38(3-4), 1540-1554. 